\title{
Grupos de Discusión y HJ-Biplot: Una Nueva Forma de Análisis Textual
}

\author{
Daniel Caballero Julia ${ }^{1}$, Purificación Vicente Galindo ${ }^{2}$, M ${ }^{\text {a }}$ Purificación Galindo \\ Villardón ${ }^{2}$
}

Daniel.caballero.sociologo@gmail.com,purivic@yahoo.com,pgalindo@usal.es

${ }^{1}$ Université Lumière Lyon 2, 4Bis Rue de l'Université, 69007 Lyon, France

${ }^{2}$ Universidad de Salamanca, Calle Alfonso X El Sabio s/n. 37007 Salamanca, España

DOI: 10.17013/risti.e2.19-35

Resumen- Este trabajo da un paso más allá en la integración de métodos cualitativos de investigación y técnicas cuantitativas analizando Grupos de Discusión desde una perspectiva multivariante con el apoyo del HJ-Biplot. Una propuesta metodológica que combina la codificación cualitativa con un nuevo valor matemático de caracterización. La propuesta se aplica al estudio de las representaciones en el discurso sobre Calidad de Vida de los ludópatas.

Palabras-chave: análisis textual; calidad de vida; ludopatía; hj-biplot; grupos de discusión.

\section{Focus Groups and HJ-Biplot: A New Way to The Textual Analysis}

Abstract - This work moves a step beyond the integration of qualitative methods of research and quantitative techniques analysing Focus groups from a multivariate perspective with the support of the HJ-Biplot. A methodological proposition that combines qualitative codification with a new mathematical characterization value in the matrix creation. The proposition is applied to the study of the representations in the discourse of the Quality of life of pathological gamblers.

Keywords: textual analysis; quality of life; pathological gambling; hj-biplot; focus groups. 


\section{Introducción}

\section{Antecedentes}

Este artículo centra su atención en el análisis textual. Este artículo muestra el trabajo que se inicia a partir de distintas líneas de trabajo anteriormente abiertas. O dicho de otra manera, este artículo introduce las últimas teorías en las que se basará. Estas serán de una parte el Análisis Estadístico de Datos Textuales (AEDT) ofrecido en (Osuna, 2006) como continuación de los trabajos de Lebart, Salem y Bécue que nos ofrecen generalmente el tratamiento de amplios textos o bases de datos con contenido textual a través de diferentes metodologías estadísticas multivariantes. Lebart, Salem, \& Bécue (2000) a partir de las diferentes posibilidades que ofrecen diversos autores, optan por el Análisis Factorial de Correspondencias (AFC) de Benzècri (1973) mientras que, por su parte, Osuna (2006) muestra algunas consideraciones y ventajas a la hora de utilizar el HJ-Biplot de Galindo (1985). Ambas opciones (AFC y HJ-Biplot) buscan la representación gráfica en dimensión reducida de una hipernube formada por la representación de un determinado número de palabras (unidades léxicas) que aparecen en una serie de documentos. Estos documentos son generalmente las respuestas a preguntas abiertas. Y de otra parte, el Análisis Cualitativo presentado en Murillo \& Mena (2006). Del lado cualitativo, se ofrece un análisis profundo de los elementos presentes, por norma general, en un discurso. Estos elementos son captados bajo la forma de códigos temáticos o conceptuales que el hablante utiliza, incluyéndose así los procesos de construcción social y del significado (Murillo \& Mena, 2006).

Ambas líneas encontrarán en este trabajo un punto de fusión gracias a la aplicación del análisis multivariante HJ-Biplot.

\section{Análisis Estadístico de Datos Textuales}

Las técnicas empleadas en el AEDT están fundamentadas en la descomposición de Valores Singulares de una matriz de datos. Esta matriz, está habitualmente compuesta por las frecuencias de las palabras de un texto (generalmente de gran tamaño o que reúne múltiples fragmentos). A esta matriz se le conoce con el nombre de Tabla Léxica.

La construcción de una Tabla Léxica es posible gracias a la aplicación de un protocolo (con una descripción más detallada en (Osuna, 2006) y (Lebart, Salem, \& Bécue, 2000) que estandariza, segmenta y lematiza el contenido de un texto. El resultado es una Tabla de Frecuencias con las repeticiones de las Unidades Léxicas (fragmentos en los que se ha dividido el texto, ya sean frases, lemas o palabras).

(Lebart, Salem, \& Bécue, 200o) expone el AEDT como un fuente cuantitativa de información. El proceso hace posible el tratamiento de grandes textos o bases de datos textuales. Esta metodología ha sido empleada para el tratamiento de preguntas abiertas de cuestionarios. Los autores se han decantado por el Análisis Factorial de Correspondencias (AFC) entre los distintos tipos de análisis estadístico multivariante.

El potencial de la familia Biplot, especialmente el HJ-Biplot para el AEDT es contrastado en (Osuna, 2006). Esta técnica muestra en un mismo gráfico las filas y columnas de una matriz en el mismo sistema de referencia y con una óptima calidad de representación para ambas. 


\section{Aproximación Cualitativa}

En contraste, las técnicas cualitativas analizan el texto (habitualmente un discurso cuando hablamos de análisis sociológicos) para extraer los elementos presentes en él. Estos elementos son codificados y presentados como unidades temáticas o conceptuales que son utilizadas por el hablante. En las técnicas cualitativas, el investigador(a) tiene en consideración la construcción social de sentido (Murillo \& Mena, 2006).

Con el apoyo de la Tabla 1 se contrasta en este artículo ambas perspectivas y técnicas e ilustra las diferencias con las cuales este estudio pretende concebir una herramienta metodológica que combine las técnicas de análisis cuantitativo y cualitativo en todos los niveles.

Así, a nivel epistemológico, las técnicas distributivas buscan los hechos (que pueden ser cuantificables), frente a las técnicas cualitativas, o estructurales, que prestan su atención al significado, a los sentidos (el sentido de las cosas, palabras o expresiones) buscados por las técnicas estructurales.

Así mismo, las técnicas cuantitativas fijan más su atención en la distribución de los fenómenos nutriéndose de la filosofía más positivista. Mientras que las cualitativas se centran en la relación entre las distintas partes con una influencia más constructivista. La teoría, dentro de la perspectiva distributiva, inicia el proceso de investigación fundamentándose en un proceso deductivo en pos de conseguir la predicción y el control de esos hechos cuya distribución se analiza. Mientras tanto, el proceso inductivo, donde los datos llevan a la teoría, orienta el proceso estructural.

Diferencias entre perspectivas. Elaborada a partir de las aportaciones de Jesús Ibáñez en Murillo y Mena (2006)

\begin{tabular}{|c|c|c|}
\hline PERSPECTIVA & $\begin{array}{l}\text { DISTRIBUTIVA } \\
\text { (Cuantitativa) }\end{array}$ & $\begin{array}{l}\text { ESTRUCTURAL } \\
\text { (Cualitativa) }\end{array}$ \\
\hline Epistemología & Hechos & Significados \\
\hline $\begin{array}{l}\text { Naturaleza del } \\
\text { conocimiento }\end{array}$ & $\begin{array}{c}\text { Analizar } \\
\text { regularidades y } \\
\text { correlaciones }\end{array}$ & $\begin{array}{l}\text { Analizar discurso y } \\
\text { su sentido social }\end{array}$ \\
\hline $\begin{array}{l}\text { Objeto de } \\
\text { análisis }\end{array}$ & $\begin{array}{c}\text { Cómo se } \\
\text { distribuyen los } \\
\text { fenómenos. } \\
\text { Positivismo }\end{array}$ & $\begin{array}{c}\text { Cómo se relacionan } \\
\text { los fenómenos. } \\
\text { Constructivismo }\end{array}$ \\
\hline Método & $\begin{array}{c}\text { Deductiva. } \\
\text { La teoría inicia el } \\
\text { proceso. } \\
\text { Predicción, } \\
\text { control. }\end{array}$ & $\begin{array}{c}\text { Inductiva } \\
\text { Los datos nos } \\
\text { llevan a la teoría. }\end{array}$ \\
\hline $\begin{array}{l}\text { Técnicas } \\
\text { Instrumentos de } \\
\text { recolección de } \\
\text { datos }\end{array}$ & $\begin{array}{c}\text { Encuesta } \\
\text { Estadísticas } \\
\text { formalizadas }\end{array}$ & $\begin{array}{c}\text { Grupos de } \\
\text { Discusión } \\
\text { Interviews } \\
\text { Historias de Vida }\end{array}$ \\
\hline
\end{tabular}


Existen también diferencias en los distintos instrumentos utilizados para la recogida de los datos, pero no entraremos en esos detalles aquí, ya que no es el objetivo de este artículo. No obstante, hay que destacar uno de los instrumentos cualitativos, ya que será el centro de nuestra atención: el Grupo de Discusión.

La naturaleza del conocimiento de esta nueva metodología debe combinar el análisis de frecuencias (palabras en nuestro caso) con el análisis de sentido social de éstas. La perspectiva cualitativa nos diría sobre esto (como el propuesto por el AEDT) que el estudio de las frecuencias mínimas puede ser incluso más importante que el estudio de las altas frecuencias.

La familia Biplot y el AFC ofrecen una buena alternativa para fusionar ambos objetos de estudio. Ambas técnicas ofrecen una representación gráfica de la distribución de las unidades en sus variables así como, al mismo tiempo, las relaciones que existen entre ellas.

No obstante, este artículo no pretende una discusión teórica y las aproximaciones deductiva/inductiva quedarán a criterio de cada investigador/a.

\section{Grupos de Discusión}

El Grupo de Discusión, herramienta fundamental en este trabajo, lo hemos definido como un "Instrumento de recogida de información discursiva, realizada en un entorno controlado, donde los componentes del grupo representan los distintos estratos de la estructura del objeto de investigación."

El grupo de discusión se fundamenta en la extracción de los significados, normalmente con un fuerte carácter social. Y que están estructurados de acuerdo a un orden o esquema de pensamiento. El grupo de discusión es una reconstrucción artificial, "en laboratorio", del discurso natural que podemos encontrar en un contexto estructural.

Con ello, se puede decir, que el AEDT tiene como ventaja la mayor sencillez en el análisis de los datos, gracias a la utilización de herramientas que trabajan con grandes matrices, e incluso pudiéndose analizar varias matrices a la vez. Sin embargo, el AEDT con el uso de dichas herramientas, pierde los elementos matizadores y de sentido que configuran el discurso que se analiza. Esto, es precisamente lo que sociológicamente más nos interesa.

Así, la perspectiva cualitativa, ajusta las unidades analíticas al propio objeto, extrayendo los significados directamente del discurso, solucionando, así, el inconveniente del AEDT, pero, mantiene una gran complejidad de análisis que puede resultar en la pérdida de la visión global y por tanto, una caída en la subjetividad.

Queda entonces clara la compatibilidad de ambas perspectivas, ya que el punto más débil de una es el más fuerte de la otra. Por tanto, encontrar una herramienta o técnica, capaz de aunar sus fuerzas, sería no solo de gran ayuda, sino que obtendría un potencial mucho mayor que cada perspectiva por separado.

De esta manera, se requiere obtener lo máximo de cada perspectiva. Es necesario poder representar los datos de la forma más simple posible (permitiéndose así su interpretación) con la máxima información de sentido posible. Por tanto, el poder de reducción del AEDT es necesario pero se requiere de la capacidad analítica de la 
perspectiva cualitativa, especialmente para captar el sentido del discurso (perdido generalmente en las representaciones del AEDT al estar centradas en las frecuencias de mayor nivel).

\section{Objetivos}

El objetivo principal de esta investigación es entonces la búsqueda de una herramienta multivariante que permita aprovechar y fusionar ambas perspectivas (cuantitativacualitativa).

Y los objetivos específicos serán:

a) Aprovechar la codificación cualitativa del objeto.

b) Aprovechar el potencial analítico de la estructura discursiva de los grupos de discusión.

c) Aprovechar la capacidad de reducción de la complejidad de las técnicas multivariantes.

d) Representar gráficamente los datos utilizando la estructura interna de los mismos.

\section{Material y Métodos}

\section{Material}

Se cuenta con el material de 5 Grupos de Discusión procedentes de la Investigación inédita cualitativa (Caballero D. , s/f) ASALJAR1. En ella, se extrajeron 32 Códigos de Sentido (entendidos como la dotación de significado) y 58 de referencia (es decir, alusiones a distintos elementos).

El tema principal de esta investigación fue la Calidad de Vida Relacionada con la Ludopatía, que fue entendida como:

"la participación de los individuos en las distintas relaciones sociales, económicas y políticas, donde la ludopatía impide una realización normal de las mismas junto con un deterioro psicológico y físico"

\section{Método}

El proceso de preparación y tratamiento de los datos parte de la transcripción literal de los grupos de discusión realizados con ASALJAR. A partir de ellos, se realizan tablas léxicas con las que obtener las frecuencias necesarias para el AEDT con las modificaciones oportunas para no olvidar ciertos requisitos cualitativos y mejorar así su aplicabilidad. Tras ello, reduciremos para hacer más operativa, la tabla léxica con la

\footnotetext{
${ }^{1}$ ASALJAR: Asociación Salmantina de Jugadores de Azar en Rehabilitación
} 
ayuda de una corrección matemática. Y finalmente utilizaremos el HJ-Biplot para analizar las tablas. Veámoslo con más detalle:

1. Transcripción y tablas de frecuencias

Las distintas transcripciones, fueron codificadas cualitativamente con la ayuda del software Atlas.ti, de una manera sistemática y rigurosa. Toda palabra, frase o párrafo debía ser incluido en alguna categoría de sentido y de referencia. Estas categorías fueron generadas a partir del sentido que daban los propios participantes, por lo que el trabajo de los codificadores era extraer dichos sentidos. Así, una vez terminado este paso previo, se solicita al mismo programa un resumen de las codificaciones para cada uno de los códigos, diferenciando aquellos que son de Sentido de los que son de Referencia. Se puede así comprobar que el texto recibe dos "pasadas" por lo que tendremos cada grupo fragmentado en los distintos códigos y duplicado (uno en Sentido y otro en Referencia).

Una vez recuperada la codificación exhaustiva de los documentos se extraen los distintos verbatims para cada uno de los códigos en cada uno de los grupos. Siguiendo las indicaciones del AEDT, nuevamente con Atlas.ti, solicitamos la creación de las tablas de frecuencias de palabras en función de los códigos dónde están presentes. Estas tablas serán después depuradas a través de un protocolo.

\section{Construcción de Tablas Léxicas}

Las Tablas Léxicas son una representación de las frecuencias de las distintas Unidades Léxicas (en este caso palabras) de los códigos (Sentido y Referencia) en cada uno de los grupos. Su creación requiere de un paso adicional: la aplicación de un protocolo2. Éste, consta de un proceso de Normalización, en el que se procura corregir errores o evitar futuras malinterpretaciones en palabras o expresiones que pueden ser ambiguas o que estén expresadas por ejemplo, en siglas; seguidamente se procede con la Segmentación en Unidades Léxicas (bien sean palabras, frases, etc.) indicando en la tabla qué frecuencias hay que considerar; y finalmente, la Lematización, donde, una vez eliminadas las palabras vacías (es decir, carentes de significado por sí mismas, como determinantes, preposiciones y similares), pasamos los verbos al infinitivo y los nombres y adjetivos al singular (el protocolo a menudo implica también pasar estos al masculino, pero para mantener la diferenciación de género esto no ha sido considerado).

El objetivo es tener una tabla donde tengamos las distintas palabras que componen el discurso (una vez filtradas por el protocolo) en las filas y los códigos (sentido o referencia) en las columnas, así para cada tipo de código y para cada grupo.

2 Más detallado en Osuna, 2006 y Lebart, Salem, \& Bécue, 2000 
Ejemplo de Tabla Léxica de los códigos de Sentido

\begin{tabular}{c|cccc} 
SENTIDO & Cod. 1 & Cod. 2 & $\ldots$ & Cod. p \\
\hline Pal. 1 & $f_{11}$ & & & $f_{1 p}$ \\
Pal. 2 & & $\ldots$ & & \\
$\ldots$ & & & & \\
Pal. n & & &.. & \\
& $f_{n 1}$ & & & \\
\end{tabular}

Ejemplo de Tabla Léxica de los códigos de Referencia

\begin{tabular}{c|cccc} 
REFERENCIAS & Cod. 1 & Cod. 2 & $\ldots$ & Cod.p \\
\hline Pal. 1 & $f_{11}$ & & & $f_{1 p}$ \\
Pal. 2 & & $\ldots$ & & \\
$\ldots$ & & & $\ldots$ & \\
Pal. $n$ & $f_{n 1}$ & & & $f_{n p}$
\end{tabular}

3. Caracterización de los datos: Valor de Caracterización

Una vez conseguida la tabla léxica, necesitamos caracterizar los datos. Es decir, necesitamos que el reparto de nuestras palabras (que son nuestras unidades léxicas) en las distintas columnas (códigos incluidos por el análisis cualitativo) se haga más evidente. Quizás éste sea el paso más importante en esta metodología y responde a la necesidad de simplificar la tabla ya que la magnitud es muy grande. La teoría nos ofrecía algunas alternativas como los perfiles, del Análisis Factorial de Correspondencias (AFC) (Benzècri, 1973), que otorga a cada casilla un peso teniendlo en consideración los marginales. Esta opción ha sido rechazada ya que dicha técnica implica, lo queramos o no, una ponderación de los datos en función de totales de fila y columna y la simple consideración de los marginales no aportaba información cualitativa relevante al análisis ni consideraba la importancia de tener en columnas códigos cualitativos. La siguiente, propuesta en (Osuna, 2006), supone buscar los valores atípicos, con la ayuda, por ejemplo, del Biplot Robusto. Pero esta opción la hemos de rechazar ya que cualitativamente algo poco frecuente puede ser sumamente importante. 
Así, proponemos lo que hemos llamado Valor de Caracterización que considera el dato de cada celda relativizada con los máximos de fila y de columna, en lugar de los marginales del AFC o la simple consideración de la cantidad. Hacer esto implica observar qué palabras son "más propias" o "más particulares" de un determinado código. O dicho de otra manera, qué palabras son las usadas dentro de una determinada unidad de sentido/referencia.

Por tanto, proponemos transformar la tabla de datos original aplicando la siguiente fórmula:

$f^{\prime}{ }_{\mathfrak{n p}}=\frac{f_{n p}}{\sqrt{\max _{i}} \sqrt{\max _{j}}}$

Transformar una tabla léxica con este procedimiento tiene los siguientes efectos:

- Los valores pierden su magnitud original y quedan acotados entre o y 1 , siendo o la ausencia de la palabra dentro de un código determinado y 1 la palabra más característica de un código.

- $\quad$ Cuando $f_{i j}$ es máximo de fila y máximo de columna: $f_{i j}=1$.

- Cuando $\mathrm{f}_{\mathrm{ij}}$ es máximo de columna, pero no de fila se relativiza el valor original con el máximo de la fila

$$
\mathrm{f}_{\mathrm{ij}}^{\prime}=\frac{\mathrm{f}_{\mathrm{ij}}}{\max _{\mathrm{i}}}
$$

- Cuando $f_{i j}$ es máximo de fila, pero no de columna, se aumenta la "caracterización del individuo (palabra) en esa variable (código), es decir, se le da más importancia (matemática) de la que originalmente tenía.

- Cuando no es ni máximo de columna, ni máximo de fila, el valor reduce su magnitud respecto del valor original. Es decir, esta palabra no es característica ni dentro de un código ni entre ellos.

Además de estas características, la aplicación del Valor de Caracterización aporta otra ventaja: la regulación de la cantidad de datos que queremos analizar. Dado que los resultados quedan expresados en valores entre o y 1 podemos observar cuál es el valor máximo que alcanza cada palabra (observando el máximo de fila de la nueva tabla), eliminando así las palabras que pueden ser muy comunes pero no alcanzan un nivel mínimo de caracterización al no ser suficientemente características de ningún código.

4. HJ-Biplot

Finalmente, una vez transformadas las Tablas Léxicas, utilizamos el HJ-Biplot (Galindo P. , 1986) (Galindo \& Cuadras, Una extensión del método Biplot y su relación con otras técnicas, 1986) una técnica de análisis estadístico multivariante que permite la representación gráfica simultánea, similar en ciertos aspectos al AFC, de la información contenida en las filas (individuos) y columnas (variables) de una matriz de datos. Se opta por esta opción, en primer lugar por su potencial y en segundo porque, teniendo las Tabla Léxica caracterizadas, aplicar el AFC, como proponían (Lebart, Salem, \& Bécue, 2000), supondría una nueva transformación a los datos, adicional a la 
que acabamos de explicar. Esto evidentemente desvirtuaría los datos al igual que las propiedades del Valor de Caracterización.

Así, el HJ-Biplot, es una representación gráfica multivariante de las líneas de una matriz Xnxp mediante marcadores $\mathrm{j} 1, \ldots . . \mathrm{jn}$ para las filas y h1,...hp para las columnas, elegidos de forma que ambos marcadores pueden ser representados en un mismo sistema de referencia con la misma calidad de representación

Para su análisis se utiliza el software Multbiplot (Vicente, 2010), que nos permite de forma rápida y sencilla incorporar nuestras tablas desde un formato *.xls generadas automáticamente por Atlas.ti3 (con las correcciones previamente vistas).

\section{Resultados}

Aplicado este método, obtenemos una tabla de sentido y otra de referencia para cada grupo. Lo que en suma supone 10 tablas:

Tabla léxica de sentido 1: 637 pals. X 29 códs.; 2: 800 pals. X 27 códs.; 3: 581 pals. X 22 códs.; 4: 628 pals. X 26 códs.; 5: 664 pals. X 27 códs.

Tabla léxica de referencias 1: 509 pals. X 40 códs.; 2: 653 pals. X 34 códs.; 3: 581 pals. X 36 códs.; 4: 475 pals. X 39 códs.; 5: 705 pals. X 45 códs.

\section{Primeros comentarios}

De esta manera, se observa que no todas las Tablas Léxicas son iguales. Cada grupo omite ciertos temas en su discurso. Cualitativamente esto tiene ya interpretación. Pues si bien es útil e importante fijarse en la información que contienen los grupos, los grupos de discusión tienen la ventaja de ofrecer una comparación entre los distintos códigos que nos invita a prestar atención también, con la misma o más importancia, a lo que no aparece. Así, los códigos ausentes en el discurso, muestran aquello que no forma parte del esquema mental del objeto o que por alguna razón, consciente o no, ha sido suprimido del discurso.

Las tablas de sentido y de referencia del primer grupo (jóvenes en primeras fases de rehabilitación) no muestran información sobre los códigos que tienen que ver con la visibilidad de la enfermedad. Al igual que se hace notar la ausencia de referencias orientadas a las relaciones de mayor distancia, tales como organismos e instituciones de nivel macro.

En las tablas de sentido y referencia del segundo grupo, enfermos más rehabilitados y de más edad, no encontramos los elementos que denotan la calidad de las relaciones, así como referencias individualizadas al igual que las relaciones más distantes o macro se pierden en gran medida. Se pierde también la consideración de uno mismo, al igual que los aspectos más físico-médicos.

El grupo tres, sin embargo, formado por los familiares de los enfermos más jóvenes, no menciona en su discurso elementos sobre la percepción social, así como las

\footnotetext{
3 (Vicente, 2010)
} 
concepciones de estigma o normalidad. Igualmente se pierden las referencias a los colectivos más distantes o dicho de otra forma, vínculos más débiles (Granovetter, 1973).

El cuarto grupo, familiares de aquellos ludópatas más rehabilitados, suprime de su discurso los elementos que podríamos llamar negativos de la calidad de vida, tales como actos ilegales, empeorar o no-normalidad, entre otros. Continuando con esta dinámica discursiva en la que no aparecen las relaciones "con uno mismo" y las más distantes, el grupo 4 también obvia en su discurso los elementos más referidos al aspecto físico-médico.

Y finalmente el quinto grupo, formado de manera mixta, no nos dice nada a cerca de estos mismos elementos más negativos sobre los actos ludópatas. Así como no hace referencia al aspecto más individualizado de las relaciones (autodirigidas) o aquellas de mayor orden en forma generalizada (otros conocidos).

Supone esto por tanto, que autopercepción y no-normalidad no está presente en ninguno de los grupos donde intervienen los familiares (GD3, 4 y 5) y podemos ver cómo tal consideración solo está en el discurso del enfermo.

\section{Resultados del análisis HJ-Biplot}

Aplicando el HJ-Biplot a nuestras tablas obtendremos lo siguiente:

En el aspecto del análisis HJ-Biplot (figura 1) sobre la tabla reducida a partir de un Valor de Caracterización mayor a 0,3 , podemos ver como tenemos una serie de códigos (vectores) en torno a los ejes y cuadrantes, y una serie de palabras que se desmarcan del gran conjunto que encontramos en el centro de gravedad. Se observa cómo la absorción total de información por parte de los ejes es de un 34,02\%.

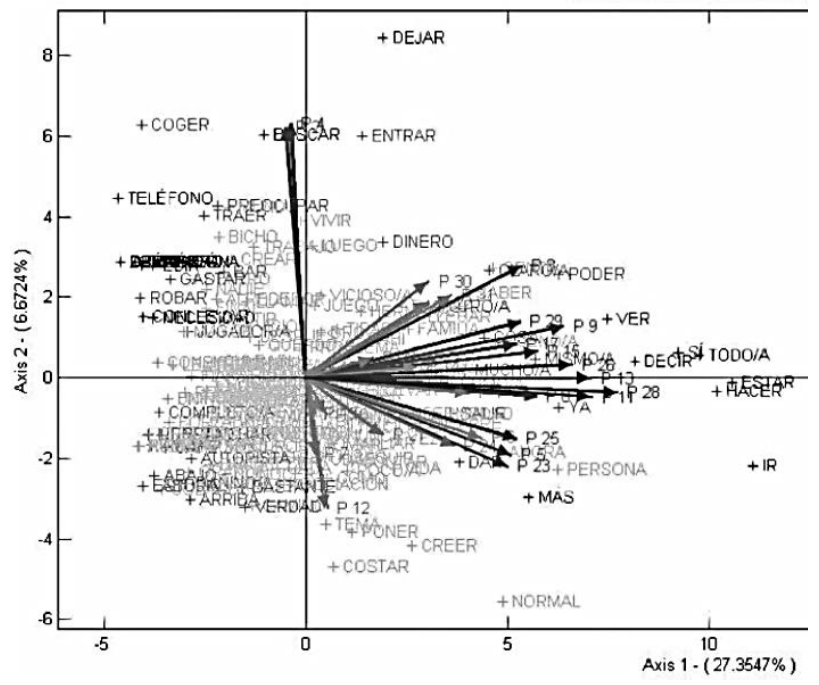

Figura 1 - HJ-Biplot. Sentido. GD 1 
Aunque hay presentes distintas calidades de representación la falta de calidad de representación sólo supondría un inconveniente a nivel matemático, ya que cualitativamente una menor frecuencia no necesariamente ha de implicar menor importancia.

La figura 2 centrando la imagen, se puede ver cómo las variables (los códigos cualitativos) representadas por vectores, se distribuyen por el plano.

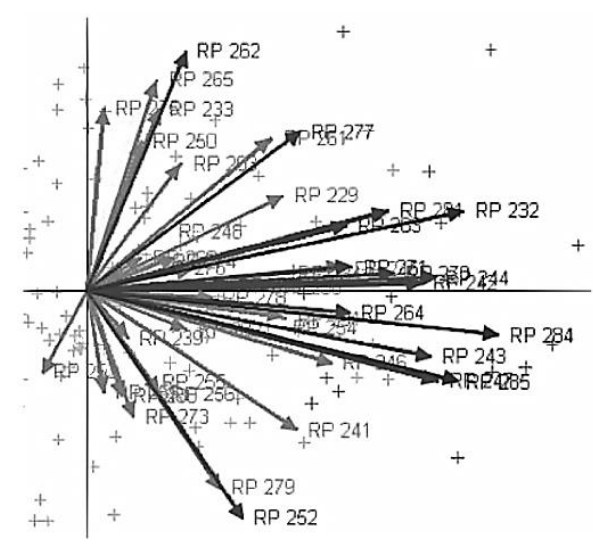

Figura 2 - HJ-Biplot Referencias Plano1-2 GD 5

Y más claramente en figuras 3 y 4 se ve que los vectores forman agrupaciones en parejas o tríos tanto en sentido como en referencias. Las altas correlaciones entre vectores, muestran la estructura del discurso, ya que se da una alta coincidencia entre los resultados Biplot y el análisis cualitativo del discurso de los grupos.

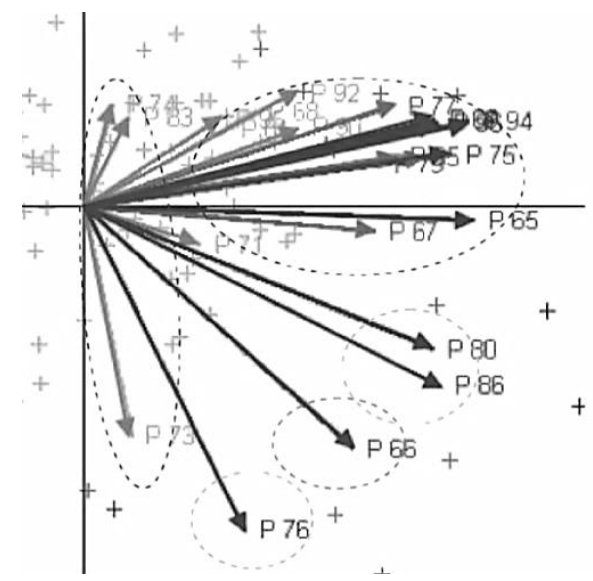

Figura 3 - HJ-Biplot Sentido Plano1-2 GD 3 


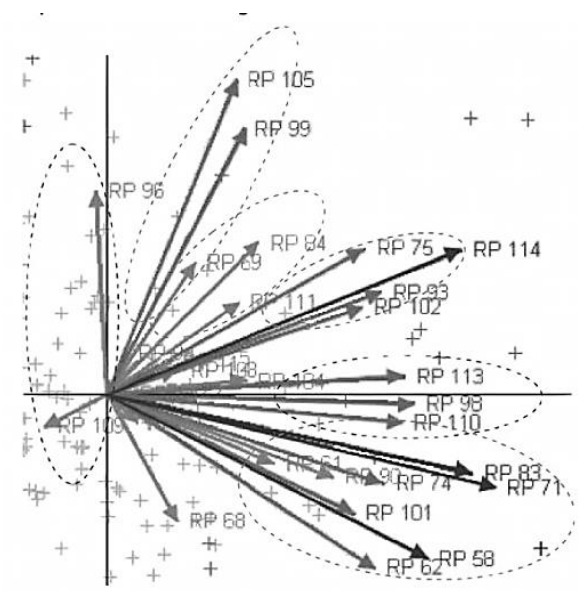

Figura 4 - HJ-Biplot Referencias Plano1-2 GD 2

Por otro lado, también se puede observar los marcadores para las palabras de las cuales extraer información (ver figura 5). Es plausible reconstruir una posible frase como "la sociedad/gente cree/entiende/comenta que el ludópata es una persona viciosa”. Viéndose además que estas palabras, se encuentran en torno a los vectores de los códigos "Vicio" y "Percepción". De igual modo se nos ofrece muestras claras de unidades léxicas relacionadas con el código en cuestión (Por ejemplo, el código Medios de Comunicación, haciendo especial hincapié en los programas de televisión "Juega y' Gana" nocturnos)

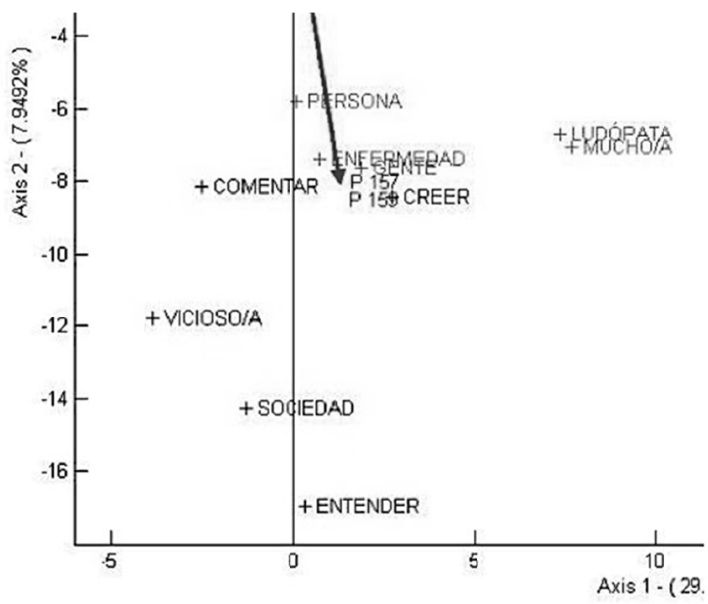

Figura 5 - HJ-Biplot Sentido Plano1-2 GD 3

Por lo tanto, la interpretación de los resultados, desde un punto de vista metodológico, guarda su interés no sólo en la distribución de las palabras en grupos o posibles frases (facultad que este análisis demuestra tener), sino que la creación de los diferentes ejes a partir de unidades léxicas pertenecientes a códigos cualitativos implica un soporte 
extra a la hora de encontrar variables latentes en el discurso generado mediante investigación con grupos de discusión.

Al mismo tiempo, la distribución de los vectores nos aporta las relaciones existentes entre ellos, pero no a partir de la interpretación del investigador sino a partir de la distribución de las unidades léxicas.

Igualmente la interpretación de los HJ-Biplot según esta técnica mantiene las características expuestas en Osuna (2006) y Lebart, Salem, \& Bécue, (2000), tales como que la longitud de los vectores son interpretables como la variabilidad léxica (riqueza) de nuestro código o que el ángulo entre vectores está relacionado con la covarianza entre los vectores, o dicho de otra manera que los discursos en esos códigos están relacionados (contienen las mismas palabras, se dan a la vez, etc.).

3. Resultados desde un punto de vista sociológico:

\section{a. Grupo 1. Sentido y Referencias}

Centrándonos en el análisis de la calidad de vida relacionada con la ludopatía, pretendida en el estudio del cual tomamos los datos, podemos apreciar como el primer eje muestra un discurso influenciado por una calidad de vida caracterizada por la enfermedad, que obstaculiza la vida y que viene acompañada de un deterioro físicomédico y social (especialmente en cuanto a la percepción que se tiene del individuo donde entran en juego la adicción y la consideración de la normalidad).

En el análisis de las referencias de este grupo, podemos ver que el discurso en el plano 1-2 queda caracterizado por las diferentes facetas de la rehabilitación donde se ven los diferentes influjos de la rehabilitación, así como de la enfermedad implicando la afectación de la familia y la mención al consumo (a menudo mención al dinero simplemente), actividades sociales e instituciones sociales (generalmente el Estado) en un tiempo posterior. El eje 2, queda definido por las relaciones con la pareja o esposa las cuales se contraponen al otro generalizado y las relaciones laborales que se tuvieran en el pasado activo. Mientras que el primer eje queda recogiendo alusiones a las relaciones centradas en el propio sujeto (el "yo" como elemento principal) ligado a un mundo social poco definido, donde apenas aparecen menciones a las relaciones de amistad, familiares lejanos o instituciones. Todo ello, en un tiempo marcado por los inicios de la rehabilitación.

\section{b. Grupo 2. Sentido y Referencias}

De un modo más narrativo, experimentado y rehabilitado, el grupo de enfermos de más edad y más tiempo en rehabilitación, muestra un plano de análisis del discurso mucho más marcado por las relaciones sociales que por los aspectos más físico-psicológicos. Además, como variable de plano encontramos la mentira junto con la variable-código empeorar. El eje 2, parece tener una clara interpretación fundamentada en el Estigma Social donde la ludopatía como enfermedad es entendida o percibida por el entorno social como un vicio.

En cuanto a las referencias del segundo grupo, se observa que las relaciones con otros familiares (a menudo refiriéndose a la familia en sentido generalizado) y los aspectos psicológicos en un época de jugador activo, junto con referencias a la asociación y algunas a los progenitores, son las partes que caracterizan a este eje. El segundo eje, 
nos muestras, el conjunto de relaciones familiares y más cercanas al grupo, en un tiempo tras un primer año sin juego. En el primero, no obstante veíamos aspectos previos a la rehabilitación.

\section{c. Grupo 3. Sentido y Referencias}

En el primer eje, encontramos la afectación en la calidad de vida del ludópata (a ojos del familiar de apoyo) a nivel física, mental y social, donde el aspecto adictivo y actitudinal son los más relevantes que viene marcada por la adicción y su asociado comportamiento que conllevan un deterioro, actos ilegales y culpa en una situación de juego y financiación encubierta por la mentira.

El plano 1-2 de las referencias, muestra elementos de las relaciones sociales dándonos una imagen de las distintas facetas y elementos de la vida como ludópata.

\section{d. Grupo 4. Sentido y Referencias}

La interpretación del sentido del discurso fundamentada en la contraposición de conceptos opuestos. Oculto/visible, apoyo/obstáculo enlazados con la opinión, la asociación y la enfermedad. Por otro lado, estigma social, percepción social y vicio se muestran más influyentes en la creación del segundo eje.

El plano de referencias muestra el proceso de transformación de las relaciones

\section{e. Grupo 5. Sentido y Referencias}

El grupo 5, que implicaba la inclusión de ludópatas y familiares de ludópatas, mostraba un importante primer eje, del cuál podíamos extraer aspectos relacionados con la aducción donde intervienen las relaciones y la asociación. En este marco, el carácter oculto de la ludopatía supone un problema (o atrae los problemas) y aparecen los obstáculos para una buena calidad de vida. Así, este grupo, caracterizando a los ludópatas (especialmente de mentirosos y solitarios) nos exponen las afectaciones físico-médicas de la ludopatía así como una expresión identitaria para con ésta.

El eje 2 por otro lado, muestra los códigos de percepción social y vicio. Confirmándose así, que, si bien los elementos con los que se presenta la calidad de vida en el discurso varían de un grupo a otro reuniéndose en torno del primer eje, el segundo eje presenta de una manera u otra el influjo del Estigma social (identidad deteriorada, percepción social de la enfermedad como vicio, etc.) sobre el colectivo ludópata.

El análisis de as referencias del quinto grupo, se caracteriza en términos generales con un primer eje fundamentado en la recogida de la orientación hacia el juego (antes y durante las primeras fases) y las relaciones con los familiares (especialmente a partir del comienzo de la rehabilitación). El segundo eje nos muestra aspectos del comienzo de la rehabilitación donde el otro generalizado, otra financiación (normalmente obtenida mediante el robo a familiares) correlacionan fuertemente con los padresmadres y el mundo mediático.

"En general, podemos ver como las referencias se orientan hacia el desarrollo de la rehabilitación, haciendo incluso referencia a ella y a la asociación, albergando, sin embargo las referencias laborales en fase anterior a éstas junto con las demás (posiblemente alguna comparación o ejemplo al hablar)" (Caballero D. , 2011, pág. 83). 
En definitiva, se han podido obtener resultados perfectamente analizables, con grandes espacios comunes en la interpretación entre las perspectivas cualitativas y cuantitativas, donde se vislumbran grandes posibilidades para la fusión entre ambas técnicas.

\section{Conclusiones}

Múltiples son las conclusiones que podemos sacar de lo aquí expuesto: sobre el trabajo, sobre los resultados y sobre la técnica en sí.

\section{Sobre el trabajo}

- El trabajo es solo una muestra de todo lo que la técnica permite.

- El trabajo muestra el éxito en la aplicación conjunta de ambas perspectivas de análisis (cuantitativa y cualitativa), aprovechando de ambas el potencial analítico.

- Se encuentran coincidencias en las conclusiones sociológicas (Cualitativas/Cuantitativas).

\section{Sobre los resultados (en relación a la CVRL)}

- En términos muy generales, el discurso entre enfermos más jóvenes y en primeras fases de rehabilitación y el resto de familiares es más parecido entre sí.

- Todos resaltan la participación de la asociación en la mejoría.

- La configuración del discurso en general, contiene en el primer eje los elementos de la CVRL mientras que el segundo eje, recoge lo que llamamos estigma.

\section{Sobre la técnica}

- La transformación propuesta de los datos, Valor de Caracterización supone un método eficaz y "modulable".

- La información que podemos extraer es una parte de toda la existente.

- No supone sociológica ni cualitativamente un problema el aceptar o incluir en la interpretación códigos mal representados ya que principalmente esto es debido a la baja variabilidad y poca frecuencia en el discurso (matemáticamente problemático pero cualitativamente importante).

- La técnica (y el HJ-Biplot) añade o aporta ligereza y vistosidad al análisis, pero pierde en riqueza y matices, sobre todo a la hora de aportar datos o ejemplo empíricos: frases, palabras, verbatims (ya que muchas se pierden). Ambas técnicas han de ir unidas. 
- Los vectores por sí solos, tanto en sentido como en referencias, aportan mucha información.

- Las palabras en torno al primer eje tienen poco valor cualitativo, ya que siguen mostrando las palabras más repetidas (sobre todo verbos como los auxiliares).

- La interpretación de las referencias es más compleja y hueca teóricamente ya que es el sentido el que aporta el relleno teórico. (Esto es coherente con su función dentro de los Grupos de Discusión).

- La técnica ha demostrado ser útil, tanto para corregir los errores vistos en las técnicas para el AEDT anterior, como para servir de gran ayuda al Análisis Cualitativo.

\section{Agradecimientos}

Al Departamento de Estadística de la Universidad de Salamanca y a todo su equipo con especial atención de su directora. Agradecer también una vez más la colaboración de la ASALJAR a la que dedicamos este artículo. Y al mismo tiempo, no puede faltar nuestro agradecimiento a los consejos de Doña Soledad Murillo, Sandra Robles y Lucía Herrarte.

\section{Referencias}

Caballero, D. (s/f). Calidad de Vida en la ludopatía: una aproximación sociológica.

Caballero, D. (3 de Noviembre de 2011). El HJ-Biplot como herramienta en el análisis de Grupos de Discusión. Calidad de Vida en la ludopatía: una propuesta sociológica. Obtenido de Repositorio Institucional de la Universidad de Salamanca Gredos: http://hdl.handle.net/10366/108778

Lebart, L., Salem, A., \& Bécue, M. (2000). Análisis Estadístico de textos. Lleida: Milenio.

Atlas.ti 5.0 de Science Plus Group BV. . (s.f.).

Benzècri, J. P. (1973). L'Analyse des Donées: L'Analyse des correspondences. Paris: Dunod.

Galindo, M. P. (1985). Contribuciones a la Representación Simultánea de Datos Muldimensionales. Tesis Doctoral. Salamanca: Universidad de Salamanca.

Galindo, M. P., \& Cuadras, C. M. (1986). Una extensión del método Biplot y su relación con otras técnicas. Publicaciones de Bioestadística y Biomatemática $\mathrm{N}^{\circ} 17$, Universidad de Barcelona.

Galindo, P. (1986). Una alternativa de representación simultanea: HJ-Biplot. Qüestiió , 13-23. 
Granovetter, M. (1983). The Strength of Weak Ties: A Network Theory Revisited. Sociological Theory 1, 201-233. doi:10.2307/202051

Murillo, S., \& Mena, L. (2006). Detectives y Camaleones: el grupo de discusión. Una propuesta para la investigación cualitativa. Madrid: Ágora.

Osuna Marín, Z. (2006). Contribuciones al Análisis de Datos Textuales. Salamanca: Universidad de Salamanca.

Vicente, J. L. (2010). MULTBIPLOT. Departamento de Estadística. Universidad de Salamanca. 